\title{
Efavirenz challenge in patients with nevirapine induced Stevens-Johnson Syndrome
}

\author{
Thandekile Cecilia Manzinia*, Bernadett Isabel Gosnella ${ }^{a}$, Melanie-Anne Amanda John ${ }^{\mathrm{b}}$ and Mahomed Yunus Suleman Moosa \\ ${ }^{a}$ Department of Infectious Diseases, University of KwaZulu-Natal, Durban, South Africa \\ ${ }^{b}$ Enhancing Care Foundation, South Africa, Department of Infectious Diseases, University of KwaZulu-Natal, Durban, South Africa \\ *Corresponding author, email: Manzini@ukzn.ac.za
}

\begin{abstract}
Background: Non-nucleoside reverse transcriptase inhibitors (NNRTI) are recommended as part of first-line treatment by the World Health Organisation (WHO) for antiretroviral treatment (ART) naïve subjects. Due to reports of cross-toxicity between nevirapine (NVP) and efavirenz (EFV), there is reluctance to substitute EFV for NVP when serious toxicity occurs. In such cases lopinavir/ritonavir (LPV/r) remains the only alternative. We report on our experience of patients with Stevens-Johnson Syndrome (SJS) secondary to NVP who were challenged with EFV under careful supervision.

Methods: A retrospective chart review of patients who presented with SJS presumed to be secondary to NVP who were challenged with EFV and were followed up for at least 2 months.

Results: The study included 13 patients. All were female, median age 28 years and median weight $66.5 \mathrm{~kg}$. The median CD4 cell count at ART initiation was 160 cells/ul. Twelve patients (92\%) developed a rash within 4 weeks of NVP. The median alanine transaminase at SJS presentation was $30 \mathrm{U} / \mathrm{l}$. The median time to EFV challenge was 30 days. The median hospital stay for SJS was 30 days and median follow up was 19 months. Eleven (85\%) patients tolerated co-trimoxazole rechallenge, two had no prior exposure. Median hospital stay for EFV challenge was additional 10 days. Twelve patients (92\%) tolerated the EFV challenge. One patient developed a pruritic maculo-papular eruption within 3 days of challenge which led to discontinuation of EFV.

Conclusion: The risk of recurrence of SJS with EFV challenge in patients with NVP induced SJS is low. It is safe to challenge patients with EFV with careful observation.
\end{abstract}

Keywords: efavirenz, nevirapine, Stevens-Johnson Syndrome

\begin{abstract}
Introduction
Stevens-Johnson syndrome (SJS) and toxic epidermal necrolysis (TEN) are considered severe cutaneous reactions to drugs, characterised by extensive detachment of the epidermis and erosions of mucous membranes. Evidence suggests that SJS and TEN are a single disease with a common pathogenesis, the main difference being the extent of cutaneous involvement. ${ }^{1}$ Drugs, including sulfonamides, allopurinol, amine anti-epileptics (phenytoin and carbamazepine), lamotrigine, and the oxicam non-steroidal anti-inflammatories are considered inciting agents and responsible for the majority of cases. However, infections such as Mycoplasma spp. and Herpes simplex virus have been implicated. This toxic effect of drugs can range from mild hypersensitivity with no mucosal involvement to severe disease with involvement of the eyes, mouth, vagina and gastrointestinal tract. SJS is associated with a mortality of $1-5 \% .^{2}$
\end{abstract}

Compared with the general population, patients with human immunodeficiency virus (HIV) have a 1000 -fold higher risk of severe skin reactions. Incidence of SJS/TEN is estimated at $1 / 1000$ per year compared to $2 / 1000000$ per year in the HIV negative population. ${ }^{3,4}$

Management of SJS includes fluid resuscitation, nutritional support, topical treatment and withdrawal of the offending drug. The causative drug needs to be substituted with an alternative. However, in some instances where options are limited, rechallenging the same drug in incremental doses may be used to desensitise against severe reactions, e.g. co-trimoxazole. ${ }^{5}$ So too for anti-tuberculous drugs that are re-introduced in a stepwise regimen to ascertain the inciting agent. ${ }^{6,7}$
Non-nucleoside reverse transcriptase inhibitors (NNRTI) are an important component of first line highly active antiretroviral treatment (HAART) regimens. They are preferred due to their efficacy, tolerability and minimal long-term toxicities. Although NVP is generally well tolerated, hepatic and cutaneous toxicities have limited their use. SJS/TEN has been observed in $0.3 \%-1 \%$ of patients on NVP, usually manifesting within the first 4-6 weeks of treatment. ${ }^{8}$ The extent of cross-toxicity between EFV and NVP is unclear. Their molecular structures are different; however, limited knowledge of the mechanism of hypersensitivity reactions precludes a definite conclusion on the risk of cross-toxicity. One review concluded that EFV should not be substituted for NVP if life threatening toxicities to NVP occur. ${ }^{9}$

In resource-limited settings few options are available when faced with NNRTI toxicity. Newer agents such as the integrase inhibitors are beyond the affordability of most resource poor settings. The only protease inhibitor (PI) freely available is lopinavir/ritonavir (Kaletra/Aluvia). The capsule formulation of this drug has several limitations including administration with food, gastrointestinal intolerance, hepatotoxicity, high pill burden, cost and limited options if resistance emerges. Hence, when faced with NVP toxicity there is good reason to consider EFV as an option rather than proceeding to LPV/r.

This study describes our experience with EFV challenge in a group of patients with NVP associated SJS.

\section{Methods}

A retrospective chart review was performed on all patients admitted to the infectious diseases unit at King Edward VIII 
Table 1: Baseline characteristics of patients with nevirapine induced Stevens-Johnson Syndrome

\begin{tabular}{|c|c|}
\hline \multicolumn{2}{|l|}{ Parameter } \\
\hline Median age, years (range) & $28(22-42)$ \\
\hline Female sex, $n(\%)$ & $13(100 \%)$ \\
\hline Median weight (range) & $66.5 \mathrm{~kg}(42-88)$ \\
\hline \multicolumn{2}{|l|}{ Time from ART to development of rash } \\
\hline$<2$ weeks & 4 \\
\hline 2-4 weeks & 9 \\
\hline 4-6 weeks & 1 \\
\hline \multicolumn{2}{|l|}{ Rash distribution, $n(\%)$} \\
\hline Mouth & $13(100 \%)$ \\
\hline Eye & $10(77 \%)$ \\
\hline Trunk & $10(77 \%)$ \\
\hline Upper limbs & $12(92 \%)$ \\
\hline Lower limbs & $11(85 \%)$ \\
\hline \multicolumn{2}{|l|}{ Concomitant medication } \\
\hline Cotrimoxazole & $11(85 \%)$ \\
\hline \multicolumn{2}{|l|}{ Opportunistic diseases at presentation } \\
\hline Kaposi sarcoma & 1 \\
\hline \multicolumn{2}{|l|}{ Laboratory findings } \\
\hline 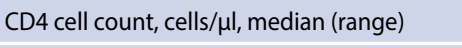 & $160(44-481)$ \\
\hline *Alanine transferase U/I, median (range) & $30.5(19-380)$ \\
\hline \multicolumn{2}{|l|}{ Hospitalisation and follow-up } \\
\hline Median length of stay for SJS, days (range) & $30(14-60)$ \\
\hline Median follow-up duration, months (range) & $19(2-36)$ \\
\hline
\end{tabular}

*Alanine transferase normal range: 10-40U/l.

hospital (KEH), Durban, South Africa, between 2004 and 2013 with a diagnosis of SJS secondary to NVP. KEH serves as a tertiary level referral centre for infectious diseases and complicated dermatologic conditions for the eastern seaboard of KwaZuluNatal province. Inclusion criteria for the study were age $\geq 18$ years, a high clinical probability of NVP-induced SJS, well documented challenge with EFV containing HAART following resolution of the rash and follow-up of at least 2 months on EFV-based HAART.

On presentation, patients were admitted under the care of a specialist dermatologist. Patients were challenged after all skin and mucosal lesions had healed. All subjects were challenged under strict supervision by an internist with daily examination for new lesions. Patients were counselled to report any cutaneous symptoms or new lesions. The study received ethics approval from the Biomedical Research Ethics Committee of University of KwaZulu-Natal (reference number BE275/12). Approval for the study was also obtained from the Department of Health and King Edward VIII Hospital.

\section{Results}

A total of 21 patients were recruited. Eight patients were excluded because it could not be clearly determined if cotrimoxazole or NVP was responsible for the SJS due to poor documentation. A total of 13 patients met the inclusion criteria. All patients were female with median age of 28 years and median follow up of 19 months. All but one developed the rash within 4 weeks of ART. Seven patients had extensive generalised lesions. The median duration of hospital stay was 36 days (Table 1).
Management of SJS was mainly supportive; and, included adequate fluid resuscitation, fluid maintenance, nutritional support, and appropriate management of the cutaneous, ocular and oral lesions with topical agents such as aqueous cream, emollients, steroid creams, mupirocin ointment, antibiotic containing eye ointments, and antiseptic oral rinsing agents. Nine patients received specific treatment for SJS, one received intravenous immunoglobulins for three days and eight (62\%) received prednisone ( $60 \mathrm{mg} /$ day) for median of five days.

Eleven patients on concomitant co-trimoxazole at onset of SJS were successfully rechallenged with co-trimoxazole, two had no previous exposure. Median time to EFV challenge was 30 days (range: 19-40 days). Median hospital stay for EFV challenge was 10 days (range: $4-22$ ).

Of the 13 patients challenged with EFV one (8\%) developed an itchy maculo-papular eruption involving the trunk and upper limbs within 3 days of challenge. ART was stopped. Once the rash resolved, this patient was placed on a LPV/r containing regimen with no further event.

\section{Discussion}

Resource limited countries have few antiretroviral agents to select from, particularly after the first line agents have been exhausted. Within the NNRTI class of drugs, EFV and NVP are readily available and affordable. With serious toxicity to one agent in this class, there is reluctance to use another for fear of cross-toxicity. ${ }^{9}$ In such a situation, one is forced to move to another class of drugs, namely $\mathrm{Pl}$, which have pharmacokinetic 
and toxicity limitations. Here we demonstrate that challenging a patient with EFV after serious NVP skin toxicity can be safely achieved under carefully controlled conditions. To our knowledge, this is the first study focussing on EFV challenge after NVP-associated SJS. Our study population was restricted to females as NVP was used almost exclusively in females of child bearing age as per our national ARV guidelines at the time of study. Most patients developed the NVP rash between two and four weeks of treatment. This is in keeping with literature where presentations of SJS typically occur within 3 weeks of drug exposure but can range from 4-28 days. ${ }^{10}$ In addition, we show that the risk of recurrence is very low on challenge with EFV. One of 13 patients (8\%) demonstrated occurrence of a new rash.

In the literature, the risk of rash recurrence with EFV in patients with moderate to severe NVP induced rash is reported at $8-25 \%$, which is in keeping with risk of recurrence in this study. ${ }^{11-13}$ Kiertiburanakul et al found that history of drug allergy and CD4 cell count $<100$ cells $/ \mu$ l at the time of NVP initiation were predictive factors for unsuccessful switching from NVP to EFV in HIV-infected patients with preceding NVP-associated skin rash. ${ }^{12}$

SJS often demands prolonged hospitalisation with reported length of hospitalisation ranging from 2 to 49 days..$^{14}$ The duration of hospitalisation here was longer (14-60 days). This was likely driven by the severity and extent of skin/mucosal involvement as a result of delayed presentation (Table 1). In addition, due to poor social circumstances of the indigent patient, discharge was often delayed until virtual complete resolution of lesions. In our study, the median time to EFV challenge was 10 days versus 20 days as reported in literature. ${ }^{12}$ However, for EFV challenge, most patients required less than 2 weeks of admission to monitor for recurrence of rash.

The characteristics of cross-toxicity between NVP and EFV varies widely in literature. WHO recommends substituting EFV for NVP for non-life-threatening NVP cutaneous reactions. ${ }^{15}$ Most clinicians are likely to substitute PI's for NVP associated SJS due to concerns about increased morbidity and mortality if SJS recurs. Our study suggests that EFV can be safely substituted for NVP with close supervision. The risk of recurrence is very low and, if a cutaneous reaction does occur, early detection and prompt discontinuation of the offending agent minimises morbidity.

In this study, about $4 \%$ of subjects had raised ALT. Although there is little literature on prevalence of hepatotoxicity in NVPassociated SJS, the rate of hepatotoxicity with NVP induced rash has been reported in up to $6 \%$ of cases. ${ }^{16}$

Our study has limitations. The retrospective nature of the study limited the quality of data available. Due to the small size of the study, we could not perform inferential statistics on risk factors for various outcomes. Selection bias could not be ruled out since only patients referred to the Department of Infectious Diseases for management were included in the study.

\section{Conclusion}

This small retrospective study suggests that EFV can be safely and successfully substituted for NVP in the majority of cases with
NVP-associated SJS. This finding is of particular value in resourceconstrained settings where there is restricted availability of different classes of drugs.

Acknowledgements - All authors thank the King Edward VIII Hospital staff and patients.

\section{References}

1. Auquier-Dunant $A$, Mockenhaupt $M$, Naldi L, et al. Correlations between clinical patterns and causes of erythema multiforme majus, Stevens-Johnson syndrome, and toxic epidermal necrolysis: Results of an international prospective study. Arch Dermatol. 2002;138(8):1019-24.

2. Harr T, French L. Toxic epidermal necrolysis and Stevens-Johnson syndrome. Orphanet J. Rare Dis. 2010;5(1):39-49. doi: 10.1186/17501172-5-39.

3. Roujeau JC, Stern RS. Severe adverse cutaneous reactions to drugs N Engl J Med. 1994;331(19):1272-85. PubMed PMID: 7794310. Epub 1994/11/10. eng.

4. Rzany B, Mockenhaupt M, Stocker U, et al. Incidence of StevensJohnson syndrome and toxic epidermal necrolysis in patients with the acquired immunodeficiency syndrome in Germany. Arch Dermatol. 1993;129(8):1059.

5. Gluckstein D, Ruskin J. Rapid oral desensitization to trimethoprimsulfamethoxazole (TMP-SMZ): Use in Prophylaxis for Pneumocystis carinii pneumonia in patients with AIDS who were previously intolerant to TMP-SMZ. Clin. Infect. Dis. 1995;20(4):849-53.

6. Kura MM, Hira SK. Reintroducing antituberculosis therapy after Stevens-Johnson syndrome in human immunodeficiency virusinfected patients with tuberculosis: role of desensitization. Int J Dermatol 2001;40(7):481-4. PubMed PMID: 11679013. Epub 2001/10/27. eng.

7. Cortese LM, Soucy DM, Endy TP. Trimethoprim/sulfamethoxazole desensitization. Ann. Pharmacother. 1996 Feb;30(2):184-6. PubMed PMID: 8835054. Epub 1996/02/01. eng.

8. Pollard RB, Robinson P, Dransfield K. Safety profile of nevirapine, a nonnucleoside reverse transcriptase inhibitor for the treatment of human immunodeficiency virus infection. Clin Ther. 1998;20(6):107192. PubMed PMID: 9916603. Epub 1999/01/23. eng.

9. Mehta $U$, Maartens $G$. Is it safe to switch between efavirenz and nevirapine in the event of toxicity? Lancet Infect. Dis. 2007;7(11):733-8.

10. Parrillo S. Stevens-Johnson syndrome and toxic epidermal necrolysis. Curr. Allergy and Asthma Rep. 2007;7(4):243-7.

11. Manosuthi W, Thongyen S, Chumpathat N, et al. Incidence and risk factors of rash associated with efavirenz in HIV-infected patients with preceding nevirapine-associated rash. HIV Med. 2006;7(6):378-82.

12. Kiertiburanakul S, Malathum K, Watcharananan S, et al. Predicting factors for unsuccessful switching from nevirapine to efavirenz in HIV-infected patients who developed nevirapine-associated skin rash. Int J STD AIDS. 2009;20(3):176-9. PubMed PMID: 19255265 Epub 2009/03/04. eng.

13. Ananworanich J, Moor Z, Siangphoe U, et al. Incidence and risk factors for rash in Thai patients randomized to regimens with nevirapine, efavirenz or both drugs. AIDS. 2005;19(2):185-92. PubMed PMID: 15668544. Epub 2005/01/26. eng.

14. Saka B, Barro-Traoré $F$, Atadokpédé FA, et al. Stevens-Johnson syndrome and toxic epidermal necrolysis in sub-Saharan Africa: A multicentric study in four countries. Int J Dermatol. 2013;52(5):575-9. PubMed PMID: 23330601. Epub 2013/01/22. eng.

15. Gilks CF, Crowley S, Ekpini R, et al. The WHO public-health approach to antiretroviral treatment against HIV in resource-limited settings. Lancet. 2016;368(9534):505-10.

16. Dieterich DT, Robinson PA, Love J, et al. Drug-induced liver injury associated with the use of nonnucleoside reverse-transcriptase inhibitors. Clin. Infect. Dis. 2004;38(Supplement 2):S80-9. 